\title{
Biomedical and Biophysical Challenges for Quantitative Microscopy
}

Watt W. Webb

Applied Physics, Cornell University, Ithaca, NY 14853

From fundamental biophysical research to biomedical diagnostics, a plethora of crucial questions challenge the experimental capabilities of "Quantitative Microscopy." However, advancing technologies and new inventions have empowered success with "impossible experiments" engendering many useful consequences including those to be reported here. However, the challenges continue to push the limits of our present capabilities. Therefore this lecture will aim to identify some of these problems and opportunities, and will venture to forecast future achievements. [1]

References:

1. This research was supported by NIH-NCRR grant P41-RR04224, NSF grant DBI-0080792, DOE grant DE-FG02-99ER62809, and grants from GlaxoSmithKline and Bio-Rad. 
An incomplete biography of the papers that Watt Webb has published with the participants in this special symposium is given below. There are at least 43 total publications from which this selection has been made.

Elson,-E-L; Webb,-W-W. (1975) Concentration correlation spectroscopy: a new biophysical probe based on occupation number fluctuations. Annu. Rev. Biophys. Bioeng, 4: 311-334. (and 12 others)

Gross,-D; Loew,-L-M; Webb,-W-W. (1986) Optical imaging of cell membrane potential changes induced by applied electric fields. Biophys. J. 50: 339-348. (and 5 others with DG)

Denk,-W; Strickler,-J-H; Webb,-W-W. (1990) Two-photon laser scanning fluorescence microscopy. Science 248: 73-76. (and 4 others)

Chandra,-S; Fewtrell,-C; Millard,-P-J; Sandison,-D-R; Webb,-W-W; Morrison,-G-H. (1994) Imaging of total intracellular calcium and calcium influx and efflux in individual resting and stimulated tumor mast cells using ion microscopy. J. Biol. Chem. 269: 15186-15194. (and 1 other)

Piston,-D-W; Masters,-B-R; Webb,-W-W. (1995) Three-dimensionally resolved NAD(P)H cellular metabolic redox imaging of the in situ cornea with two-photon excitation laser scanning microscopy. J. Microsc. 178:20-27. (and 2 others)

Xu,-C; Zipfel,-W; Shear,-J-B; Williams,-R-M; Webb,-W-W. (1996) Multiphoton fluorescence excitation: new spectral windows for biological nonlinear microscopy. Proc. Natl. Acad. Sci. USA 93: 10763-10768. (and 7 others)

Brust-Mascher,-I; Webb,-W-W. (1998) Calcium waves induced by large voltage pulses in fish keratocytes. Biophys. J. 75: 1669-1678. (and 1 other)

Brown,-E-B; Shear,-J-B; Adams,-S-R; Tsien,-R-Y; Webb,-W-W. (1999) Photolysis of caged calcium in femtoliter volumes using two-photon excitation. Biophys. J. 76: 489-499. (and 2 others)

Thompson,-R-E; Larson,-D-R; Webb,-W-W. (2002) Precise nanometer localization analysis for individual fluorescent probes. Biophys. J. 82: 775-783 (first one) 